\title{
Malignant Nasopharyngeal Neoplasm
}

National Cancer Institute

\section{Source}

National Cancer Institute. Malignant Nasopharyngeal Neoplasm. NCI Thesaurus. Code C9321.

A primary or metastatic malignant neoplasm involving the nasopharynx. 